\title{
Arithmetic Method of Double-Injection-Electrode Model for Resistivity Measurement Through Metal Casing
}

\author{
Qing Chen, David Pardo, Hong-bin Li, Fu-rong Wang, and Qi-zheng Ye
}

\begin{abstract}
Through-casing resistivity (TCR) measurement instruments such as Cased Hole Formation Resistivity are extensively used for the dynamic monitoring of oil reservoirs during the production phase in oil wells to evaluate the residual oil distribution. However, two shortcomings still exist in the common TCR model based on single-injection electrodes: The real value of steel-casing resistance is difficult to acquire, and the effect from mechanical tolerances of electrode scale is unpredictable. This paper proposes an innovative model based on doubleinjection electrodes. In this new model, all the required variables can be measured simultaneously; furthermore, a compensating arithmetic method is employed to obtain the real casing resistance. Self-adaptive goal-oriented $h p$-finite-element simulations have been performed to prove that the influence of mechanical tolerances of electrode scale can be reduced effectively. Therefore, the TCR measurement accuracy is highly improved.
\end{abstract}

Index Terms-Arithmetic, electromagnetic analysis, geophysical measurements.

\section{INTRODUCTION}

D URING the last two decades, interest in electrical logging through casing has grown considerably. The idea of acquiring through-casing resistivity (TCR) measurements was first proposed by Alpin in 1939 [1]. He said that, when current is injected into a casing, the voltage differences on the casing well are influenced by the formation resistivities. Thus, by measuring the voltage differences, formation resistivities can be inferred. His method was not implemented at that time since existing logging instruments were not advanced enough to measure correctly the weak voltage signals below $1 \mu \mathrm{V}$ obtained in TCR. As a result of inadequate technology, the original patent by Alpin has laid dormant for over 30 years.

Manuscript received November 11, 2008; revised April 26, 2009 and May 26, 2009. First published September 15, 2009; current version published December 23, 2009. The work of D. Pardo was supported in part by the Spanish Ministry of Science and Innovation under Projects MTM2008-03541 and TEC2007-65214. The work of F. R. Wang was supported in part by the Research Foundation for Outstanding Young Teachers of China University of Geosciences (Wuhan) under Grant CUGQNL0840.

Q. Chen, H.-B. Li, and Q.-Z. Ye are with the College of Electrical and Electronic Engineering, Huazhong University of Science and Technology, Wuhan 430074, China (e-mail: WFR101@163.com; lihongbin@mailhust.edu. cn; yqziq@mail.hust.edu.cn).

D. Pardo is with the Basque Center for Applied Mathematics, 48170 Zamudio, Spain (e-mail: dzubiaur@yahoo.es).

F.-R. Wang is with China University of Geosciences, Wuhan 430074, China (e-mail:wfr777@163.com).

Digital Object Identifier 10.1109/TGRS.2009.2026741
Recent advances in weak-signal processing and measurement technologies allowed Alpin's method to be applied. The report on the field test of the prototype of the tool [5] was another important step in the development of the technology. The vertical resolution of the measurement, effects caused by the cement sheath, casing inhomogeneities, and finite length of the casing have been studied by Schenkel $(1990,1994)$, Kaufman (1990, 1993), Vail (1995), Tabarovsky (1994), Zinger (1994), and Singer (1995, 1998) [2]-[10].

Based on Kaufman measurement mode, various attempts have been made to build systems for logging formation resistivity in boreholes with casing [11], [12]. Computing simulation systems based on finite-element methods (FEMs) provide enough engineering suggestions to the improvements of TCR instruments [13]-[15]. Nowadays, resistivity logging samples such as Cased Hole Formation Resistivity (Schlumberger) have been applied successfully in production wells for the dynamic monitoring of oil pools and the distribution of the residual oil.

There are still two major potential problems in the most popular resistivity measurement model based on single-injection electrodes (SIEs).

1) The real value of steel casing is hard to achieve. The theoretical calculation method lacks accuracy and cannot compensate the influences of steel-casing corrosion and temperature variation. The practical measurement method provides the integral resistance of the parallel steel casing and formation that cannot correctly reflect the real value of casing, and the difference will deteriorate with the formation capacitance increasing.

2) In order to protect the measurement accuracy of the formation resistivity, the mechanical tolerances of the electrode scale will be limited to a very small range, which is difficult to carry out in practical operation. It is still not certain that the error can be controlled below 5\% even if the mechanical tolerance is below $0.01 \%$, because the influence grade is sensitive to depth and the errors will rapidly increase when the electrodes are located at the top part of the steel casing.

An innovative model based on two electrodes is recommended in this paper. The recommended model differs from the SIE model in that an accessional current injection electrode is used. An arithmetic method on how to obtain real casing resistivity and eliminate the influences caused by electrodescale tolerances will be discussed. 


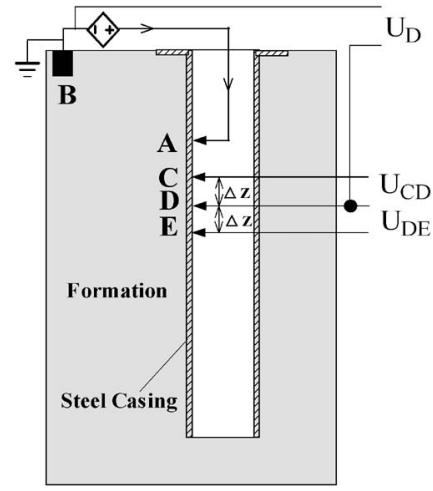

Fig. 1. SIE model.

\section{SIE MODEL}

In the view of the SIE model, the casing is assumed to be a uniform and highly conductive steel pipe with an infinite length, and the formation can be assumed to be a homogeneous medium around the casing. The leakage current is perpendicular to the casing. As shown in Fig. 1, the current is injected into the casing from electrode $\mathrm{A}$, and the formation apparent resistivity around point $\mathrm{D}$, namely, $\rho_{a}$, can be represented as

$$
\begin{aligned}
\rho_{a} & =(\Delta z)^{2} \frac{U_{D}}{\Delta^{2} U} r_{c} \\
\Delta^{2} U & =U_{\mathrm{CD}}-U_{\mathrm{DE}}
\end{aligned}
$$

where $r_{c}$ is the casing resistance per meter, $\Delta z$ is the length unit of electrode scale, and $U_{D}, U_{\mathrm{CD}}$, and $U_{\mathrm{DE}}$ are the voltage difference signals shown in Fig. 1. In fact, the computed result $\rho_{a}$ achieved from (1) is not the real resistivity of formation but the formation resistance of the horizontal layer with thickness $\Delta z$ equal to $1 \mathrm{~m}$. The conversion factor from $\rho_{a}$ to the real formation resistivity depends on the characteristic mechanical parameters of the casing.

In order to get $\rho_{a}, r_{c}$ and $\left(U_{\mathrm{CD}}-U_{\mathrm{DE}}\right)$ are indispensable besides $\Delta z$ and $U_{D}$, which are easy to measure directly.

\section{A. How to Calculate $r_{c}$}

There are two ways to calculate $r_{c}$, which means the resistance per meter of steel casing.

One is through the theoretical calculation method. Assuming known values of $\rho_{c}$ (steel-casing resistivity) and $a$ and $\Delta a$ (radius of casing and the thickness of casing, respectively), $r_{c}$ can be represented as

$$
r_{c}=\rho_{c} \frac{1}{2 \pi a \Delta a} .
$$

The corrosion of steel casing may reduce $\Delta a$ greatly. Moreover, $\rho_{c}$ is sensitive to temperature change, and a $100{ }^{\circ} \mathrm{C}$ temperature variation will bring an excursion over $20 \%$ to $\rho_{c}$. Thus, the calculated $r_{c}$ cannot reflect the real steel-casing resistance, and the theoretical calculation method will inevitably produce great error in resistivity measurement.

The other method is the practical measurement method that is immune to steel-casing corrosion and temperature variation.

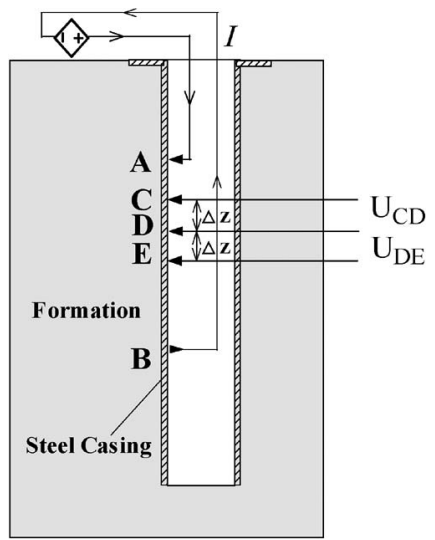

Fig. 2. $r_{c}$ measurement in the SIE model.

A connection method different from that in voltage signal, i.e., $U_{D}$ and $\Delta^{2} U$, measurement is applied, and then, the operation program will get more complex, which will lower the logging efficiency. As shown in Fig. 2, the current $I$ is injected from electrode $\mathrm{A}$ and collected at electrode $\mathrm{B}$, and $r_{c}$ can be inferred from the following:

$$
r_{c}=\frac{U_{\mathrm{CD}}}{I \Delta z}=\frac{U_{\mathrm{DE}}}{I \Delta z} .
$$

However, the result derived from (3) is not the real steelcasing resistance per meter but the integrative resistance of steel casing and formation in $1 \mathrm{~m}$. Lower formation resistivity will bring greater error.

\section{B. Influence of Mechanical Tolerances}

As shown in Fig. 1, the lengths $l_{\mathrm{CD}}$ and $l_{\mathrm{DE}}$ are required to be absolutely equal in the SIE model. It is a fact that mechanical tolerances are unavoidable in practical logging operations.

Assuming that

$$
\begin{aligned}
& l_{\mathrm{CD}}=\Delta z-\Delta l \\
& l_{\mathrm{DE}}=\Delta z+\Delta l
\end{aligned}
$$

the influences caused by mechanical tolerances $\Delta l$ are estimated in the following:

$$
\begin{aligned}
\Delta^{2} U & =U_{\mathrm{CD}}-U_{\mathrm{DE}}=r_{c} \Delta z\left(I_{\mathrm{CD}}-I_{\mathrm{DE}}\right) \\
\Delta^{2} U^{\prime} & =\Delta^{2} U-r_{c} \Delta l\left(I_{\mathrm{CD}}+I_{\mathrm{DE}}\right) .
\end{aligned}
$$

From (5) and (6), we find that

$$
e^{\prime}=\frac{\Delta^{2} U^{\prime}-\Delta^{2} U}{\Delta^{2} U}=-\frac{\Delta l\left(I_{\mathrm{CD}}+I_{\mathrm{DE}}\right)}{\Delta z\left(I_{\mathrm{CD}}-I_{\mathrm{DE}}\right)} .
$$

$I_{\mathrm{CD}}$ means the average current flowing in casing $l_{\mathrm{CD}}$ while $I_{\mathrm{DE}}$ means that in casing $l_{\mathrm{DE}}$. The sum of leakage currents from point $\mathrm{C}$ to point $\mathrm{E}$, which is equal to $\left(I_{\mathrm{CD}}-I_{\mathrm{DE}}\right)$, is far less than the current $I_{\mathrm{CD}}$ (or $I_{\mathrm{DE}}$ ) flowing along the casing. Even if the mechanical tolerance is limited to a level below $0.01 \%$, that means $\Delta l / \Delta z$ is less than $10^{-4}$, it is not certain that the system error $e^{\prime}$ can be controlled below $5 \%$. 


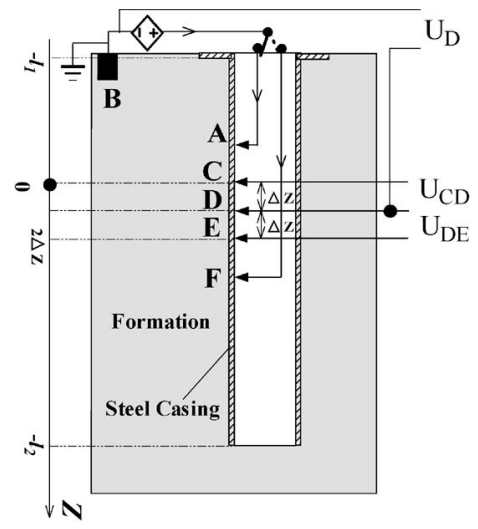

Fig. 3. DIE model.

\section{DIE MODEL}

As shown in Fig. 3, the double-injection-electrode (DIE) model differs from that of SIE in that an additional current injection electrode $\mathrm{F}$ is used and point $\mathrm{D}$ is assumed to be the midpoint of $l_{\mathrm{AF}}$. The current is injected from electrodes $\mathrm{A}$ and $\mathrm{F}$ alternately, and the injected currents are $I_{A}$ and $I_{F}$, respectively. Setting point $C$ as the origin of axis $z$, the leakage currents are described as follows:

$$
\begin{aligned}
& \int_{-l_{1}}^{0} \Delta i_{A}(z) d z=K_{1} I_{A} \\
& \int_{0}^{2 \Delta z} \Delta i_{A}(z) d z=K_{2} I_{A} \\
& \int_{2 \Delta z}^{l_{2}} \Delta i_{A}(z) d z=K_{3} I_{A} \\
& \int_{-l_{1}}^{0} \Delta i_{F}(z) d z=K_{1} I_{F} \\
& \int_{0}^{2 \Delta z} \Delta i_{F}(z) d z=K_{2} I_{F} \\
& \int_{2 \Delta z}^{l_{2}} \Delta i_{F}(z) d z=K_{3} I_{F} \\
& K_{1}+K_{2}+K_{3}=1
\end{aligned}
$$

where $\Delta i_{A}(z)$ and $\Delta i_{F}(z)$ are the leakage current distribution functions, $l_{1}$ is the distance from the top point of the casing to the origin point, $l_{2}$ is the distance from point $\mathrm{C}$ to the casing bottom, and $K_{1}, K_{2}$, and $K_{3}$ are positive constants.

\section{A. Current Distribution Functions}

Usually, the leakage current is considered flowing uniformly into the formation in the SIE model. The leakage current $\Delta i$ is relative to depth, and the variation will get more obvious with increasing formation conductance. It is easy to approximate the current distribution with quadratic functions along the casing in the DIE model

$$
\begin{aligned}
& i_{A}(z)=I_{A}\left(a_{1} z^{2}+b_{1} z+c_{1}\right) \\
& i_{F}(z)=I_{F}\left(a_{2} z^{2}+b_{2} z+c_{2}\right) .
\end{aligned}
$$

The known conditions are

$$
\begin{aligned}
i_{A}(0) & =\left(K_{2}+K_{3}\right) I_{A} \\
i_{A}(2 \Delta z) & =K_{3} I_{A} \\
i_{F}(0) & =-K_{1} I_{F} \\
i_{F}(2 \Delta z) & =-\left(K_{1}+K_{2}\right) I_{F} .
\end{aligned}
$$

Coefficients $a_{1}$ and $a_{2}$ are supposed to be

$$
-a_{1}=a_{2}=a^{*}>0 .
$$

The solutions of (9) are

$$
\begin{aligned}
& b_{1}=\frac{-K_{2}}{2 \Delta z}+2 a^{*} \Delta z \\
& c_{1}=K_{2}+K_{3} \\
& b_{2}=\frac{-K_{2}}{2 \Delta z}-2 a^{*} \Delta z \\
& c_{2}=-K_{1} .
\end{aligned}
$$

The flowing current along the casing varies with the second power of $z$ in the DIE model, while the leakage current is considered uniform in the SIE model. Theoretically, the current distribution functions would be expressed as higher order functions in terms of $z$, if more electrodes are used and more voltage differences are measured. Then, the logging system will subsequently become more complex.

\section{B. Solutions of $r_{c}, U_{D}$, and $\Delta^{2} U$}

In the DIE model, an additional connection method for casing resistivity measurement, as shown in Fig. 3, is unnecessary, and $r_{c}, U_{D}$, and $\Delta^{2} U$ can be obtained at the same time, which greatly improves the logging efficiency.

When the current $I_{A}$ is injected from electrode A, the relative voltage differences are

$$
\begin{aligned}
U_{A-\mathrm{CD}} & =r_{c} \int_{0}^{\Delta z} i_{A}(z) d z \\
U_{A-\mathrm{DE}} & =r_{c} \int_{\Delta z}^{2 \Delta z} i_{A}(z) d z \\
U_{A-\mathrm{CE}} & =U_{A-\mathrm{CD}}+U_{A-\mathrm{DE}} \\
\Delta^{2} U_{A} & =U_{A-\mathrm{CD}}-U_{A-\mathrm{DE}} .
\end{aligned}
$$

Substituting (12) into (13)

$$
\begin{aligned}
\frac{U_{A-\mathrm{CE}}}{r_{c} I_{A}} & =-\frac{4}{3} a^{*}(\Delta z)^{3}+\left(K_{2}+2 K_{3}\right) \Delta z \\
\frac{\Delta^{2} U_{A}}{r_{c} I_{A}} & =\frac{K_{2} \Delta z}{2} .
\end{aligned}
$$


$U_{F-\mathrm{CE}}$ and $\Delta \Delta U_{F}$ can be inferred in the same way as

$$
\begin{aligned}
\frac{U_{F-\mathrm{CE}}}{r_{c} I_{F}} & =\frac{4}{3} a^{*}(\Delta z)^{3}-\left(K_{2}+2 K_{1}\right) \Delta z \\
\frac{\Delta^{2} U_{F}}{r_{c} I_{F}} & =\frac{K_{2} \Delta z}{2}
\end{aligned}
$$

where $I_{F}$ is the current injected from electrode $\mathrm{F}$.

If $K_{2} \rightarrow 0$, then

$$
\begin{aligned}
\frac{K_{2} \Delta z}{2} & \cong 0 \\
\frac{4}{3} a^{*}(\Delta z)^{3} & \cong 0 .
\end{aligned}
$$

Moreover, if $K_{2} \rightarrow 1$, then

$$
\begin{aligned}
& U_{A-\mathrm{DE}} \cong 0 \\
& U_{F-\mathrm{CD}} \cong 0 \\
& U_{A-\mathrm{CE}}=\Delta^{2} U_{A}=U_{A-\mathrm{CD}} \\
& U_{F-\mathrm{CE}}=-\Delta^{2} U_{F}=U_{F-\mathrm{DE}} .
\end{aligned}
$$

Therefore, $r_{c}$ can be described as

$$
r_{c}=\frac{\Delta^{2} U_{A}+U_{A-\mathrm{CE}}}{2 I_{A} \Delta z}+\frac{\Delta^{2} U_{F}-U_{F-\mathrm{CE}}}{2 I_{F} \Delta z} .
$$

$\Delta^{2} U_{A}$ and $\Delta^{2} U_{F}$ provide the apparent compensations for the real steel-casing resistance, through which the influence of the parallel formation resistance is eliminated, particularly when the formation resistance is low.

In the innovative DIE model, the other two important parameters $U_{D}$ and $\Delta^{2} U$ presented in (1) are defined as

$$
\begin{aligned}
U_{D} & =\frac{U_{A-\mathrm{CE}} U_{F-D}-U_{F-\mathrm{CE}} U_{A-D}}{U_{A-\mathrm{CE}}-U_{F-\mathrm{CE}}} \\
\Delta^{2} U & =\frac{U_{A-\mathrm{CE}} \Delta^{2} U_{F}-U_{F-\mathrm{CE}} \Delta^{2} U_{A}}{U_{A-\mathrm{CE}}-U_{F-\mathrm{CE}}} .
\end{aligned}
$$

The selection of $U_{A-\mathrm{CE}}$ and $U_{A-\mathrm{CE}}$ as the coefficients to compute $U_{D}$ and $\Delta \Delta U$ will be discussed in Section III-C.

The final formation resistivity is given as

$$
\begin{array}{r}
\rho_{a}=\frac{\Delta z}{2}\left(\frac{\Delta^{2} U_{A}+U_{A-\mathrm{CE}}}{I_{A}}+\frac{\Delta^{2} U_{F}-U_{F-\mathrm{CE}}}{I_{F}}\right) \\
\cdot \frac{U_{A-\mathrm{CE}} U_{F-D}-U_{F-\mathrm{CE}} U_{A-D}}{U_{A-\mathrm{CE}} \Delta^{2} U_{F}-U_{F-\mathrm{CE}} \Delta^{2} U_{A}} .
\end{array}
$$

\section{Error Estimation of Mechanical Tolerances}

Equations (4)-(7) are used for references in the analysis on the error caused by mechanical tolerance $\Delta l$ in DIE model.

Supposing that the injected currents $I_{A}$ and $I_{F}$ are equal, which can be realized easily in practical resistivity logging operations

$$
I_{A}=I_{F}=I
$$

the ratio $r_{c} / \rho_{a}$ is usually close to zero, and the leakage current distribution is considered uniform

$$
a_{1}=-a_{2}=-a^{*}=0 .
$$

If $l_{\mathrm{CD}}=l_{\mathrm{DE}}=\Delta z$, then

$$
\Delta^{2} U=r_{c} I \frac{K_{2} \Delta z}{2}
$$

and if $l_{\mathrm{CD}}=\Delta z-\Delta l$ and $l_{\mathrm{DE}}=\Delta z+\Delta l$, then

$$
\Delta^{2} U^{*} \approx r_{c} I \frac{K_{2}}{2}\left[\Delta z+2 \Delta l-\frac{(\Delta l)^{2}}{\Delta z}\right] .
$$

Therefore

$$
e^{*}=\frac{\Delta^{2} U^{*}-\Delta^{2} U}{\Delta^{2} U} \approx 2 \frac{\Delta l}{\Delta z}-\left(\frac{\Delta l}{\Delta z}\right)^{2} .
$$

Comparing (7) with (25), an important conclusion is drawn: The influence caused by mechanical tolerances in the innovative DIE model is far less than that in the SIE model. It is proved that the arithmetic presented in (19) approximately eliminates the potential part of errors that is sensitive to mechanical tolerances.

\section{Simulations}

While most analytical methods cannot be applied to complex geometries, a simulation of a TCR measurement tool via numerical methods is rather challenging due to the high electrical conductivity contrast and small thickness of casing [13]-[15]. Here, we utilize a 2-D axially symmetric numerical method based on a self-adaptive goal-oriented $h p$-FEM that accurately simulates such logging measurements. This method automatically constructs an optimal grid with varying element sizes $h$ and polynomial orders of approximation $p$ throughout the computational grid, and it produces high-accuracy solutions that we employ to compare the performance of the SIE model versus that of the DIE model.

\section{A. SIE Model}

As shown in Fig. 1, the current is injected from electrode A. All the relative parameters are described as

$$
\begin{aligned}
h & =100 \mathrm{~m} \\
h_{D} & =50 \mathrm{~m} \\
l_{\mathrm{AD}} & =1.5 \mathrm{~m} \\
l_{\mathrm{CD}} & =\Delta z-\Delta l \\
l_{\mathrm{DE}} & =\Delta z+\Delta l \\
\Delta z & =0.5 \mathrm{~m} \\
a & =0.1 \mathrm{~m} \\
\Delta a & =0.01 \mathrm{~m} .
\end{aligned}
$$

In the aforementioned equation, the following can be observed: 1) $h, a$, and $\Delta a$ are the length, radius, and thickness of the casing; 2) $h_{D}$ is the distance from the ground to electrode $\mathrm{D}$; 3) $l_{\mathrm{AD}}, l_{\mathrm{CD}}$, and $l_{\mathrm{DE}}$ are the distances between $\mathrm{A}$ and $\mathrm{D}, \mathrm{C}$ 
and $\mathrm{D}$, and $\mathrm{D}$ and $\mathrm{E}$, respectively; 4) $\Delta z$ is the length unit of electrode scale, and it is equal to $0.5 \mathrm{~m}$; and 5) $\Delta l$ is the mechanical tolerance. The casing resistivity $\rho_{c}$, the borehole resistivity $\rho_{b}$, and the formation resistivity $\rho_{a}$ are assumed to be $1 \times 10^{-6}, 1$, and $1 \Omega \cdot \mathrm{m}$, respectively.

If there is no mechanical tolerance, $\Delta l$ is equal to zero. Setting the injected current $I_{A}$ as $100 \mathrm{~A}$, the potentials at electrodes A, C, D, and E can be calculated

$$
\begin{aligned}
& U_{A}=69.2907533457684 \mathrm{~V} \\
& U_{C}=1.141703407631423 \mathrm{~V} \\
& U_{D}=1.137938173656035 \mathrm{~V} \\
& U_{E}=1.134214302162464 \mathrm{~V} .
\end{aligned}
$$

Substituting all the parameters of (26) and (27) into (1) and (2), the apparent formation resistivity will be obtained

$$
\rho_{1}=1.095 \Omega \cdot \mathrm{m}
$$

which is close to the real value $1 \Omega \cdot \mathrm{m}$.

If there is $10 \%$ mechanical tolerance, $\Delta l$ equals $0.05 \mathrm{~m}$. Setting the injected current $I_{A}$ as $100 \mathrm{~A}$, the potentials at electrodes A, C, D, and E can be calculated

$$
\begin{aligned}
& U_{A}=69.2772263877734 \mathrm{~V} \\
& U_{C}=1.141325424878497 \mathrm{~V} \\
& U_{D}=1.137938585076513 \mathrm{~V} \\
& U_{E}=1.133844591457561 \mathrm{~V} .
\end{aligned}
$$

Substituting all the parameters of (26) and (29) into (1) and (2), the apparent formation resistivity will be obtained

$$
\rho_{2}=-0.064 \Omega \cdot \mathrm{m} \text {. }
$$

The simulations show that $10 \%$ mechanical tolerance may bring serious measurement error to the SIE model.

\section{B. DIE Model}

As shown in Fig. 3, the current is injected now from electrodes $\mathrm{A}$ and $\mathrm{F}$ alternatively.

If there is no mechanical tolerance, $\Delta l$ is equal to zero. Setting the injected current $I_{A}$ as $100 \mathrm{~A}$, the potentials at electrodes A, C, D, and E can be calculated

$$
\begin{aligned}
U_{A} & =69.2907533457684 \mathrm{~V} \\
U_{A-C} & =1.141703407631423 \mathrm{~V} \\
U_{A-D} & =1.137938173656035 \mathrm{~V} \\
U_{A-E} & =1.134214302162464 \mathrm{~V} .
\end{aligned}
$$

Setting the injected current $I_{F}$ as $100 \mathrm{~A}$, the potentials at electrodes F, C, D, and E can be calculated accordingly

$$
\begin{aligned}
U_{F-C} & =1.134207994856528 \mathrm{~V} \\
U_{F-D} & =1.137929547892143 \mathrm{~V} \\
U_{F-E} & =1.141692463041506 \mathrm{~V} \\
U_{F} & =69.2855133577332 \mathrm{~V} .
\end{aligned}
$$

Substituting all the parameters of (26), (31), and (32) into (20), the apparent formation resistivity can be obtained

$$
\rho_{3}=1.036 \Omega \cdot \mathrm{m}
$$

which is close to the real value $1 \Omega \cdot \mathrm{m}$.

If there is $10 \%$ mechanical tolerance, $\Delta l$ is equal to $0.05 \mathrm{~m}$. Setting the injected currents $I_{A}$ and $I_{F}$ both as $100 \mathrm{~A}$, the potentials of electrodes A, C, D, E, and F can be calculated

$$
\begin{aligned}
U_{A} & =69.2907533457684 \mathrm{~V} \\
U_{A-C} & =114.1325424878497 \mathrm{~V} \\
U_{A-D} & =113.7938585076513 \mathrm{~V} \\
U_{A-E} & =113.3844591457561 \mathrm{~V} \\
U_{F-C} & =113.4580214238326 \mathrm{~V} \\
U_{F-D} & =113.7931466786438 \mathrm{~V} \\
U_{F-E} & =114.2072964321923 \mathrm{~V} \\
U_{F} & =69.2855133577332 \mathrm{~V} .
\end{aligned}
$$

Based on (20), the apparent formation resistivity can be calculated

$$
\rho_{4}=1.046 \Omega \cdot \mathrm{m} .
$$

The calculated results, i.e., $\rho_{3}$ and $\rho_{4}$, are very close to the assumed real formation resistivity.

We conclude that even a $10 \%$ mechanical tolerance has very little impact on the DIE logging model, while the SIE model is very sensitive to the mechanical tolerances.

To show the robustness of the DIE method, we consider again our previous models but with a new formation resistivity equal to $100 \Omega \cdot \mathrm{m}$ and a new casing resistivity equal to $2.3 \times 10^{-7} \Omega \cdot \mathrm{m}$. The relative apparent resistivities we obtain for the SIE and DIE models are

$$
\begin{aligned}
114.444 \Omega \cdot \mathrm{m}, & \Delta l=0 \mathrm{~m} ; \text { SIE method } \\
109.500 \Omega \cdot \mathrm{m}, & \Delta l=0 \mathrm{~m} ; \text { DIE method } \\
-5.619 \Omega \cdot \mathrm{m}, & \Delta l=0.05 \mathrm{~m} ; \text { SIE method } \\
110.595 \Omega \cdot \mathrm{m}, & \Delta l=0.05 \mathrm{~m} ; \text { DIE method. }
\end{aligned}
$$

Again, we observe a superior accuracy of the DIE method with respect to the SIE method.

\section{CONCLUSION}

An innovative model based on two electrodes has been recommended in this paper. Numerical results obtained with a self-adaptive goal-oriented $h p$-FEM have illustrated the performance of the new DIE model. It was proved to have three outstanding advantages over the SIE model.

1) All the relative parameters $r_{c}, U_{D}$, and $\Delta^{2} U$ can be achieved at the same time without changing the connection method.

2) A compensating arithmetic method is employed to reduce the influence of parallel formation resistance in calculating casing resistance. It is proved that the compensation has good performance whether the formation resistivity is low or high. 
3) An arithmetic method is constructed to calculate the differential voltage. The compensation arithmetic method based on the DIE model is proved to greatly reduce the errors caused by mechanical tolerances of electrode scale.

\section{REFERENCES}

[1] L. M. Alpin, "The method for logging in cased wells," U.S.S.R. Patent 56 026, Nov. 30, 1939.

[2] C. J. Schenkel and H. F. Morrison, "Effects of well casing on potential field measurements using downhole current sources," Geophys. Prospect., vol. 38, no. 6, pp. 663-686, 1990.

[3] A. A. Kaufman, "The electrical field in the borehole with the casing," Geophysics, vol. 55, no. 1, pp. 29-38, Jan. 1990.

[4] A. A. Kaufman, "A transmission-line model for electrical logging through casing," Geophysics, vol. 58, no. 12, pp. 1739-1747, Dec. 1993.

[5] W. B. Vail, S. T. Momii, and R. Woodhouse, "Through casing resistivity tool TM to locate bypassed oil," Amer. Oil Gas Report., vol. 38, no. 11, pp. 70-76, 1995.

[6] C. J. Schenkel and H. F. Morrison, "Electrical resistivity measurement through metal casing," Geophysics, vol. 59, no. 7, pp. 1072-1082, Jul. 1994.

[7] L. A. Tabarovsky, M. E. Cram, T. V. Tamarchenko, K. M. Straack, and B. S. Zinger, "Through-casing resistivity (TCR): Physics, resolution and 3-D effects," in Proc. 35th Annu. Logging Symp., Soc. Petrophys., Well Log Anal., 1994, pp. TT1-TT14.

[8] B. S. Zinger, K.-M. Strack, and L. T. Tabarovsky, "Modeling of electrical effects of borehole casing inhomogeneities," in Proc. 64th Annu. Int. Mtg., Soc. Explor. Geophys., Expanded Abstr., 1994, pp. 399-402.

[9] B. S. Singer, "Through-casing resistivity: 2-D and 3-D distortions and correction techniques," in Proc. 36th Annu. Logging Symp., Soc. Petrophys., Well Log Anal., 1995, pp. 1-12.

[10] B. S. Singer and K. M. Strack, "New aspects of through-casing resistivity theory," Geophysics, vol. 63, no. 1, pp. 52-63, Jan./Feb. 1998.

[11] S. M. Sheinmann, "On stabilization of electrical fields in the earth," Pricladnaya Geophyzika, vol. 3, pp. 3-15, 1947. (in Russian).

[12] W. B. Vail, "Electronic measurement apparatus movable in a cased borehole and compensation for casing resistance differences," U.S. Patent 5075 626, Dec. 24, 1991.

[13] D. Pardo, "Simulation of multifrequency borehole resistivity measurements through metal casing using a goal-oriented hp finiteelement method," IEEE Trans. Geosci. Remote Sens., vol. 44, no. 8, pp. 2125-2134, Aug. 2006.

[14] H. Wang, "Simultaneous reconstruction of geometric parameter and resistivity around borehole in horizontally stratified formation from multiarray induction logging data," IEEE Trans. Geosci. Remote Sens., vol. 41, no. 1, pp. 81-89, Jan. 2003.

[15] M. J. Nam, H. J. Kim, Y. Song, T. J. Lee, and J. H. Suh, "Threedimensional topographic and bathymetric effects on magnetotelluric responses in Jeju Island, Korea," Geophys. J. Int., vol. 176, no. 2, pp. 457466, Feb. 2009.

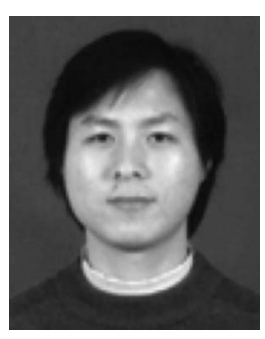

Qing Chen was born in Zhongxiang, China, in 1979. He received the B.S., M.S., and Ph.D. degrees from the College of Electrical and Electronic Engineering, Huazhong University of Science and Technology (HUST), Wuhan, China, in 2002, 2005, and 2008, respectively.

He is currently a Postdoctoral Worker with the College of Electrical and Electronic Engineering, HUST. His research is mainly focused on innovative sensing methods and weak-signal-detection technologies.

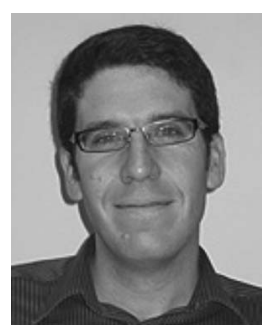

David Pardo received the B.S. degree in mathematics from the University of the Basque Country, Bilbao, Spain, in 2000 and the M.S. and Ph.D. degrees in computational and applied mathematics from The University of Texas, Austin, in 2002 and 2004, respectively.

Then, he was a Postdoctoral Fellow and a Research Associate with the Petroleum and Geosystems Engineering, The University of Texas, during the period 2004-2008. Since September 2008, he has been a Research Professor and the Team Leader of the Basque Center for Applied Mathematics, Zamudio, Spain. His research interests include computational electromagnetics, petroleum-engineering applications (borehole simulations), adaptive finite-element methods, multigrid solvers, and multiphysics and inverse problems.

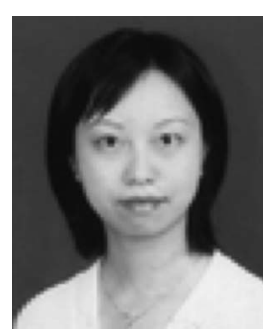

Hong-bin Li was born in Changsha, China, in 1967. She received the Ph.D. degree from the Huazhong University of Science and Technology (HUST), Wuhan, China, in 1994.

Since 1994, she has been a Faculty Member with the College of Electrical and Electronic Engineering, HUST, where she is currently a Professor. Her research interests include highly accurate measurement for weak current or voltage signals, data processing and error analysis of experimental systems, and so on.

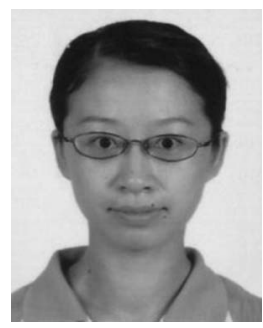

Fu-rong Wang received the B.S. degree in petroleum engineering and the M.S. and Ph.D. degrees in mineral resource exploration and prospecting from the China University of Geosciences (CUG), Wuhan, China, in 2001, 2004, and 2007, respectively.

Then, she became a Lecturer with CUG. Her major research interests include reservoir sedimentary, mineral exploration, and oil-well logging.

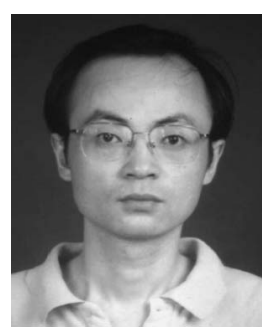

Qi-zheng Ye was born in Wuhan, China, in 1965. He received the B.S. degree in physics from Hubei University, Wuhan, in 1986, the M.S. degree in optical instruments from the Changchun Institute of Optics, Fine Mechanics and Physics, Chinese Academy of Science, Changchun, China, in 1991, and the Ph.D. degree in electrical engineering from the Huazhong University of Science and Technology (HUST), Wuhan, in 2001

Since 2004, he has been a Professor with the College of Electrical and Electronic Engineering, HUST. His main research interests include electromagnetism, electrical discharge, and plasma techniques in environmental engineering. He has published over 50 journal papers in these areas. 